\section{EXCELLENT IMAGE QUALITY}

The Orthophos XG 3D, from Sident Dental Systems, allows dentists to simultaneously perform prosthetic and surgical planning of implants and take advantage of either a laboratory or CEREC-produced highprecision drilling template. The time required for planning and production of the template is minimal for CEREC users; consequently patients will appreciate the need for treatment more readily and will value the additional safety offered by templateguided implantation.

Incorporating the latest 3D imaging technology, the Orthophos XG 3D delivers excellent image quality, minimum radiation dose, intuitive software and efficient clinical workflow. It enables the operator to switch between 2D panoramic and 3D imaging at the touch of a button. Optimised for day-to-day practice assignments it incorporates height adjustable field of view options for maximum efficiency. It enables clinicians to see more detail and treat patients more efficiently because it provides more diagnostic benefits and better treatment planning capabilities.

Its 3D capabilities are ideal for implant procedures enabling implantologists to generate radiographs, carry out in-depth diagnostics, treatment plan and produce surgical guides.

Call 01932582900 or email j.colville@sident.co.uk.

\title{
ACCURATE MEASUREMENT OF THE ROOT CANAL
}

Quality Endodontic Distributors Ltd are UK distributors of the Morita Root ZX Mini.

Compact and lightweight, the Root ZX Mini facilitates accurate measurement of the root canal, requires no zero adjustment, and incorporates an automatic calibration facility and a clear liquid crystal display. It supplements the existing Dentaport System and the Tri Auto ZX.

The compact Root ZX Mini apex locator is battery operated, light, tough and easily transported. Featuring a reflector-type colour liquid crystal screen and piezo-electric beeper it makes accurate measurements whether the canal is dry or wet. File posi- tion and movement are clearly shown in real time with easy to read increments for high precision measurements. The display instantly changes to green as the file approaches the apex. File position is also indicated by the audible signal for additional precision. Easy to use, the Root ZX Mini can memorise two types of working length.

Telephone QED on 01733 404999. www.qedendo.co.uk

\section{EXEMPLARILY QUIET COMPRESSORS}

Dürr Dental are renowned for the sturdiness, durability and performance of their equipment. Now they've further enhanced their Tornado compressors making both the one and two cylinder systems not only exemplarily quiet but their energy consumption particularly efficient.

The new Tornado 1 and Tornado 2 combine all the advantages of their predecessor models: dental air of the highest quality, a compact form, excellent sturdiness and lasting value. An optional, pre-assembled, noise-reducing hood subtly emphasises the award-winning design of the Tornado. Together with an elaborate air extraction solution it also operates extremely quietly at an incredible $54 \mathrm{~dB}$, which is comparable to the noise level of a warm summer rain shower or a whispered conversation!

These attributes not only spare ears and nerves; the new Tornados also work substantially more efficiently than earlier models and require 15\% less energy for the same performance. The units can be supplied with a membrane dryer if required and this enables uninterrupted operation around the clock. At the same time the systems guarantee the highest standards of hygiene, among other things, through the use of an anti-bacterial inner coating on the pressure tanks.

These features mean that Tornado compressors are powerful and economic enough to supply up to three treatment rooms.

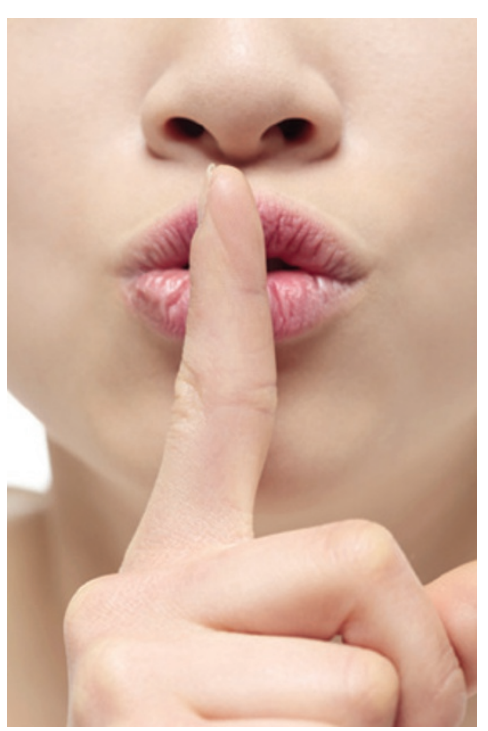

\section{EASY HANDLING AND PRECISE IMAGING}

Sirona is expanding its digital impression product line. The new digital impressioning system APOLLO DI features easy handling coupled with precise imaging at a low cost.

The APOLLO DI features easy handling, precise imaging, and the proven Sirona Connect workflow. The impression system includes an imaging unit, APOLLO Connect software, and the APOLLO DI intraoral camera, with which users can make digital impressions of the clinical situation in a seamless workflow. To do this, a moisture-insensitive highcontrast spray is sprayed on the teeth very finely. Fine particles in the spray ensure high contrast and thus very precise images.

The Sirona product line now includes the CEREC Bluecam, the CEREC Omnicam, and the APOLLO DI. Although these three cameras with their different imaging methods are tailored to the different needs of various dental practices, the interaction between the practice and the lab is the same - the digital model data can be quickly and easily transmitted to the dental laboratory.

Call Sirona Dental Systems on 08450715040 . 